OPEN ACCESS

Edited by:

Georgios Karanikolos, Khalifa University,

United Arab Emirates

Reviewed by:

Konstantinos Triantafyllidis,

Aristotle University of

Thessaloniki, Greece

Md A. Wahab,

Chengdu University, China

*Correspondence: Apostolos Enotiadis

aenotiadis@gmail.com

Dimitrios Gournis

dgourni@uoi.gr

Petra Rudolf

p.rudolf@rug.nl

tThese authors have contributed equally to this work

Specialty section:

This article was submitted to Green and Sustainable Chemistry,

a section of the journal

Frontiers in Chemistry

Received: 22 May 2020

Accepted: 12 August 2020

Published: 17 September 2020

Citation:

Thomou E, Diamanti EK, Enotiadis A,

Spyrou K, Mitsari E, Boutsika LG,

Sapalidis A, Moretón Alfonsín E, De

Luca O, Gournis D and Rudolf $P$ (2020) New Porous Heterostructures Based on Organo-Modified Graphene

Oxide for $\mathrm{CO}_{2}$ Capture.

Front. Chem. 8:564838.

doi: 10.3389/fchem.2020.564838

\section{New Porous Heterostructures Based on Organo-Modified Graphene Oxide for $\mathrm{CO}_{2}$ Capture}

\author{
Eleni Thomou ${ }^{1,2 \dagger}$, Evmorfia K. Diamanti ${ }^{1,2 \dagger}$, Apostolos Enotiadis ${ }^{3 *}$, Konstantinos Spyrou ${ }^{1}$, \\ Efstratia Mitsari ${ }^{1}$, Lamprini G. Boutsika ${ }^{3}$, Andreas Sapalidis ${ }^{3}$, Estela Moretón Alfonsín ${ }^{2}$, \\ Oreste De Luca ${ }^{2}$, Dimitrios Gournis ${ }^{1 *}$ and Petra Rudolf ${ }^{2 *}$ \\ ${ }^{1}$ Department of Materials Science and Engineering, University of loannina, loannina, Greece, ${ }^{2}$ Zernike Institute for Advanced \\ Materials, Faculty of Science and Engineering, University of Groningen, Groningen, Netherlands, ${ }^{3}$ National Center for \\ Scientific Research "Demokritos", Athens, Greece
}

In this work, we report on a facile and rapid synthetic procedure to create highly porous heterostructures with tailored properties through the silylation of organically modified graphene oxide. Three silica precursors with various structural characteristics (comprising alkyl or phenyl groups) were employed to create high-yield silica networks as pillars between the organo-modified graphene oxide layers. The removal of organic molecules through the thermal decomposition generates porous heterostructures with very high surface areas $\left(\geq 500 \mathrm{~m}^{2} / \mathrm{g}\right)$, which are very attractive for potential use in diverse applications such as catalysis, adsorption and as fillers in polymer nanocomposites. The final hybrid products were characterized by X-ray diffraction, Fourier transform infrared and $\mathrm{X}$-ray photoelectron spectroscopies, thermogravimetric analysis, scanning electron microscopy and porosity measurements. As proof of principle, the porous heterostructure with the maximum surface area was chosen for investigating its $\mathrm{CO}_{2}$ adsorption properties.

Keywords: organosilica, graphene oxide, pillaring, hybrid structures, porous heterostructures, sorbents, $\mathrm{CO}_{2}$ capture

\section{INTRODUCTION}

The dramatic effects of global warming (sea level rise, wildfires, flooding, extreme weather conditions) and the constant degradation of our planet are among the most important and challenging issues the modern world is facing. Approximately 35 billion metric tons of $\mathrm{CO}_{2}$ are emitted globally each year (National Academies of Sciences Medicine, 2019) and with the already existing technology only an insignificant fraction of these emissions is currently prevented from contributing to the greenhouse gas effect. Instead of being considered a detrimental waste product, carbon dioxide can become a precious and perfectly sustainable source material, which, once captured, can be utilized in a plethora of commercial processes such as enhanced oil recovery, chemical or biological conversion, food industry, mineral carbonation etc. (National Academies of Sciences Medicine, 2019). Therefore the development of low cost, efficient, easily applied, reusable and environmentally friendly materials capable of capturing and storing greenhouse gases, and more specifically $\mathrm{CO}_{2}$, which constitutes a major factor in global warming, is more urgent than ever before. 
Adsorption that involves binding of carbon dioxide onto the surface of a solid sorbent, is one of the most promising $\mathrm{CO}_{2}$ separation technologies that are currently being developed and used (Leung et al., 2014). A wide range of sorbents has been proposed, from activated carbon, to zeolites, alumina, or MOFs-just to name a few (Shi et al., 2020) and among the materials that have been studied for this purpose is also graphene oxide (dos Santos and Ronconi, 2017; Huang and Feng, 2018; Pokhrel et al., 2018; Shrivastava et al., 2018). Graphene-based materials hold a prominent position as they combine a series of significant advances such as excellent physicochemical properties, high specific surface areas, low adsorption energy, high selectivity and light weight (Novoselov et al., 2012). Graphene oxide (GO), a layered structure produced by the treatment of graphite with strong oxidizing agents and characterized by a high concentration of diverse oxygencontaining functional groups, which make it hydrophilic, has been identified as an excellent host matrix for diverse functional molecular structures. Organic molecules, inorganic pillars or metal ions can be accommodated in its interlayer space to design porous hybrid materials for energy (Enotiadis et al., 2012), environmental (Duan et al., 2017) and sorption (Pedrielli et al., 2018) applications. An essential step for realizing such nanostructures was the modification/intercalation of graphene oxide with primary aliphatic chains in order to create an organophilic GO derivative that can be readily dispersed in polar organic solvents (Bourlinos et al., 2003).

Large specific surface area and high selectivity are two of the most important characteristics a material should have in order to be considered a suitable candidate for $\mathrm{CO}_{2}$ adsorption applications (Leung et al., 2014). Saha and Kienbaum showed that higher selectivity for $\mathrm{CO}_{2}$ over other gases can be achieved by introducing selected functionalities and heteroatoms (nitrogen, oxygen, sulfur) to the sorbent's surface (Saha and Kienbaum, 2019). However, it is quite challenging to meet the high specific surface area criteria when employing GO for the development of sorbents since the big disadvantage of layered materials like GO is their lack of permanent porosity: a normal layered material can for example swell upon hydration, but collapses again after dehydration. Hence, in order to overcome this obstacle, taking advantage of intercalation chemistry, permanent pillars have to be introduced between the layers in order to create a robust 3-D network of adjacent graphene sheets with nanopores of the right size and surface properties to accommodate $\mathrm{CO}_{2}$.

Pillaring of 2-D layered materials allows for a fine control of the structural characteristics of the resulting micro- and nanoporous composites (Ohtsuka, 1997) and assures structural stability, permanent pore sizes and high surface areas. Such rational interlayer design has opened new prospects for applications in areas as diverse as the nanocomposites themselves (Nicotera et al., 2011; Enotiadis et al., 2013; Zapata et al., 2013), namely catalysis (Kloprogge et al., 2005; Gil et al., 2008), metal uptake (Balomenou et al., 2008), sensors (Tonlé et al., 2007), environmental remediation (Zhao et al., 2011), supercapacitors (Yan et al., 2012; Ke and Wang, 2016; Banda et al., 2019) and Lithium-ion batteries (Hu et al., 2019).
In cases where an improved structural stability was crucial, graphene oxide sheets were kept apart via the creation of metaloxide networks in the interlayer space (Matsuo et al., 2005). Many studies have been published regarding the pillaring of graphenebased materials based on the incorporation of different silicon sources mainly by the sol-gel method. Organosilanes are silicon sources that have already been used successfully for the synthesis of periodic mesoporous organosilicas (PMOs) in the form of thin films (Wahab and He, 2009; Wahab et al., 2009) and fibers (Wahab et al., 2006), as well as materials with other morphologies depending on the choice of precursor and the experimental conditions (Wahab et al., 2004a,b, 2005). Matsuo's group has studied extensively the synthetic conditions for pillared GO with various silylating reagents such as 3-aminopropylethoxysilanes or alkyl trichlorosilane with various alkyl lengths (Matsuo et al., 2007, 2009). A pyrolysis step is required for these hybrids to obtain large surface areas and controlled pore sizes, both essential characteristics to extend their use to applications in the fields of hydrogen storage (Matsuo et al., 2012b), catalysts (Maruyama et al., 2014; Rana et al., 2015), electrodes (Yoo et al., 2011) and sensors (Duan et al., 2017).

Matsuo et al. have shown that the BET surface area of porous graphene heterostructures can be increased up to $756 \mathrm{~m}^{2} / \mathrm{g}$ after insertion of two different organosilanes between graphene layers in a two-step process (Matsuo et al., 2012a). The same group also showed that repeating silylation process of graphene oxide with trichlorosilane affects the density of the siliceous pillars, and allows to tailor the BET surface area between 77 and $723 \mathrm{~m}^{2} / \mathrm{g}$ (Matsuo et al., 2015).

In this work, a new effective and efficient silylation process is proposed for the development of high surface area materials, which is considerably faster than the ones reported in the literature so far (Matsuo et al., 2012a; Maruyama et al., 2014). The silylation method was performed on an organically modified GO derivative employing three distinct silica precursors with different structural characteristics and chosen to develop silica networks within the interlayer space of the organo-modified GO. The final products were fully characterized with a combination of techniques and the degree of silylation of each reagent was evaluated. The one bridged with a phenyl group showed the maximum amount of silica content in the final heterostructure. Subsequent pyrolysis was found to create the desired porous structure raising the samples' specific surface area up to $550 \mathrm{~m}^{2} / \mathrm{g}$. For this hybrid the potential as sorbent of $\mathrm{CO}_{2}$ was briefly explored.

\section{EXPERIMENTAL SECTION}

\section{Materials}

Graphite (purum, powder $\leq 0.2 \mathrm{~mm})$, Nitric acid $\left(65 \% \mathrm{HNO}_{3}\right)$ and Potassium chlorate $\left(\mathrm{KClO}_{3}, 98+\%\right)$ were purchased from Fluka Inc. Dodecylamine (DA, $\geq 99 \%), 1,4$-bis(triethoxysilyl)benzene (BTB 99\%), tetraethylorthosilicate (TEOS 98+\%) and (3-aminopropyl)triethoxysilane (APTEOS $\geq 98 \%$ ) were acquired from Sigma-Aldrich, and sulfuric acid $\left(\mathrm{H}_{2} \mathrm{SO}_{4}, 95-97 \%\right)$, nbutanol and sodium hydroxide $(\mathrm{NaOH})$ were obtained from Merck. All reagents were of analytical grade and used without 
<smiles>CCO[Si](CCCN)(OCC)OCC</smiles>

APTEOS<smiles>CCO[Si](OCC)(OCC)c1ccc([Si](OCC)(OCC)OCC)cc1</smiles>

BTB<smiles>CCO[Si](OCC)(OCC)OCC</smiles>

TEOS

FIGURE 1 | Chemical structures of the silica precursors used (APTEOS, BTB, and TEOS).

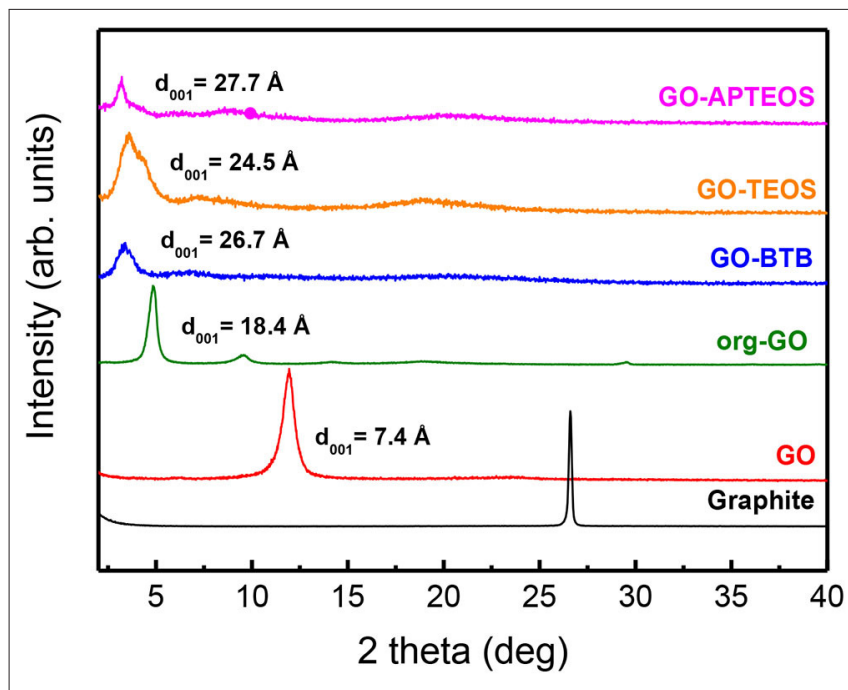

FIGURE 2 | XRD patterns of pristine graphite, graphene oxide, organo-modified GO (org-GO), and heterostructures prepared by silylation with the three different organosilane precursors: BTB, TEOS, and APTEOS (GO-BTB, GO-TEOS, GO-APTEOS).

further purification. Distilled deionized water was used for all the experiments.

\section{Graphene Oxide Synthesis}

Graphene oxide, denoted as GO in the following, was synthesized using a modified Staudenmaier's method (Staudenmaier, 1898; Gengler et al., 2010; Stergiou et al., 2010). In a typical synthesis, $10 \mathrm{~g}$ of powdered graphite was added to a mixture of $400 \mathrm{~mL}$ of $95-97 \% \mathrm{H}_{2} \mathrm{SO}_{4}$ and $200 \mathrm{~mL}$ of $65 \% \mathrm{HNO}_{3}$, while cooling in an ice-water bath in order to counteract the heat released during the very exothermic chemical reaction. $200 \mathrm{~g}$ of powdered $\mathrm{KClO}_{3}$ was added to the mixture in small portions under continuous stirring and cooling. The reaction was quenched after $18 \mathrm{~h}$ by pouring the mixture into distilled water and the oxidation product was washed until the $\mathrm{pH}$ reached an almost neutral value $(\sim 6.0)$, and finally air-dried at room temperature after being spread on a glass plate.

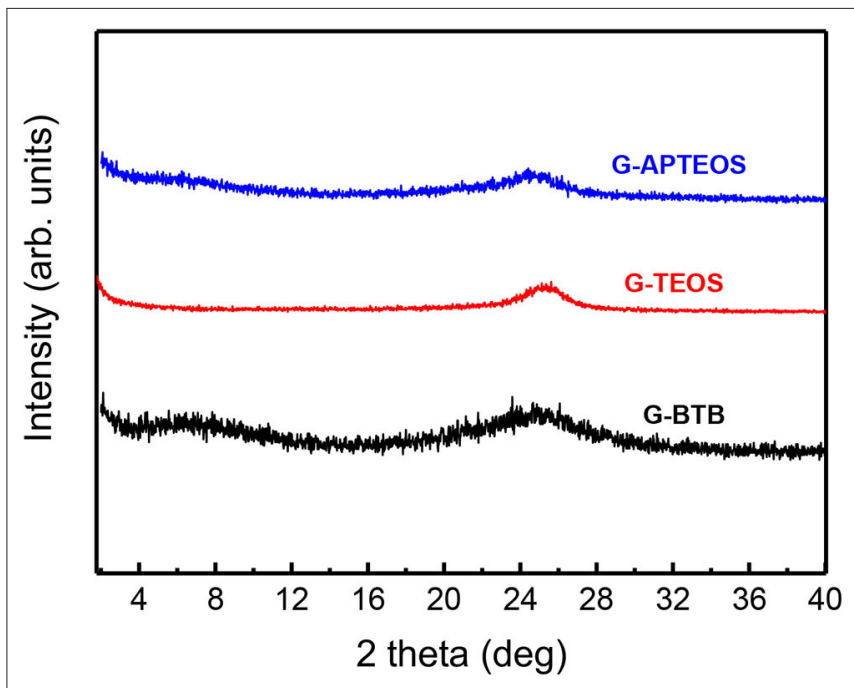

FIGURE 3 | XRD patterns of the heterostructures obtained after calcination of GO-APTEOS, GO-TEOS and GO-BTB at $370^{\circ} \mathrm{C}$ to yield G-APTEOS, G-TEOS and G-BTB.

\section{Organo-Modified Graphene Oxide}

$1.5 \mathrm{~g}$ of dodecylamine were dissolved in $50 \mathrm{~mL}$ of ethanol and the solution was added slowly to an aqueous GO suspension (beforehand $0.1 \mathrm{M} \mathrm{NaOH}$ was added to adjust the $\mathrm{pH}$ value to 7.5) under vigorous stirring (dodecylamine/GO 3:1 w/w). The mixture was stirred for $24 \mathrm{~h}$, centrifuged, washed three times with ethanol/ water: $1 / 1$, and air-dried after being spread on a glass plate. The organo-modified GO is denoted as org-GO.

\section{Silica-GO Heterostructures}

Before silylation, GO was dried a second time under vacuum at room temperature overnight. $100 \mathrm{mg}$ of org-GO were dispersed in n-butanol $(5 \mathrm{~mL})$, sonicated for $30 \mathrm{~min}$ and left under stirring overnight. The silica precursor (APTEOS, BTB or TEOS; chemical structures shown in Figure 1) was slowly added to the org-GO dispersion under stirring for $2 \mathrm{~h}$ before adding the water (containing a drop of hydrochloric acid) while keeping constant the molar ratio silica precursor/ $\mathrm{H}_{2} \mathrm{O} / \mathrm{n}$-butanol: 1/4/54. 


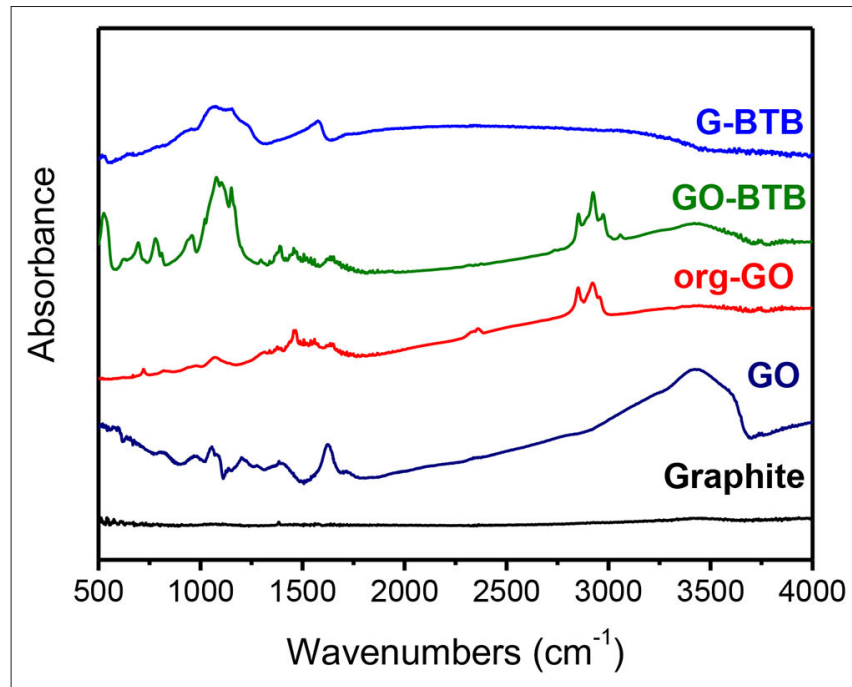

FIGURE 4 | FTIR spectra of the heterostructure prepared by silylation of organo-modified graphene oxide with 1,4-Bis(triethoxysilyl)-benzene before (GO-BTB) and after (G-BTB) calcination; the spectra of graphite, pristine graphene oxide $(\mathrm{GO})$ and graphene oxide intercalated with dodecylamine (org-GO) are plotted for comparison.

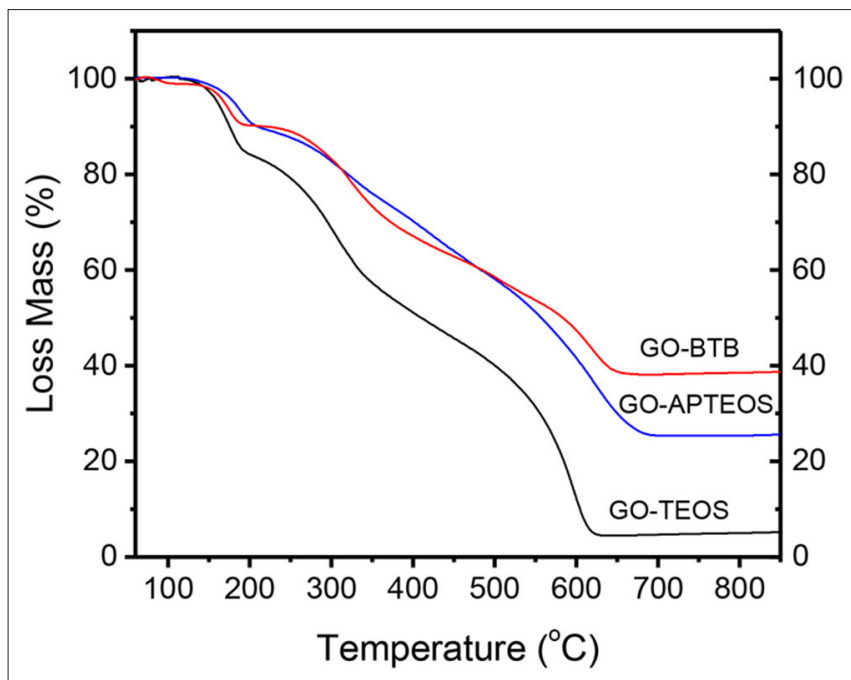

FIGURE 5 | TGA curves of heterostructures prepared by silylation of organo-modified graphene oxide with 1,4-Bis(triethoxysilyl)-benzene (GO-BTB), with (3-aminopropyl)triethoxysilane (GO-APTEOS) and with tetraethylorthosilicate (GO-TEOS).

The sol-gel reactions were performed at $50^{\circ} \mathrm{C}$ while stirring, and the obtained gel was placed in the oven at $50^{\circ} \mathrm{C}$ overnight. The silica-GO organo-heterostructures are denoted as GOBTB, GO-APTEOS and GO-TEOS, depending on which of the three organosilica precursors was employed. The final porous structures were collected after calcination in air at $370^{\circ} \mathrm{C}$ for $120 \mathrm{~min}$. The calcinated samples are denoted as G-BTB, GAPTEOS, and G-TEOS.

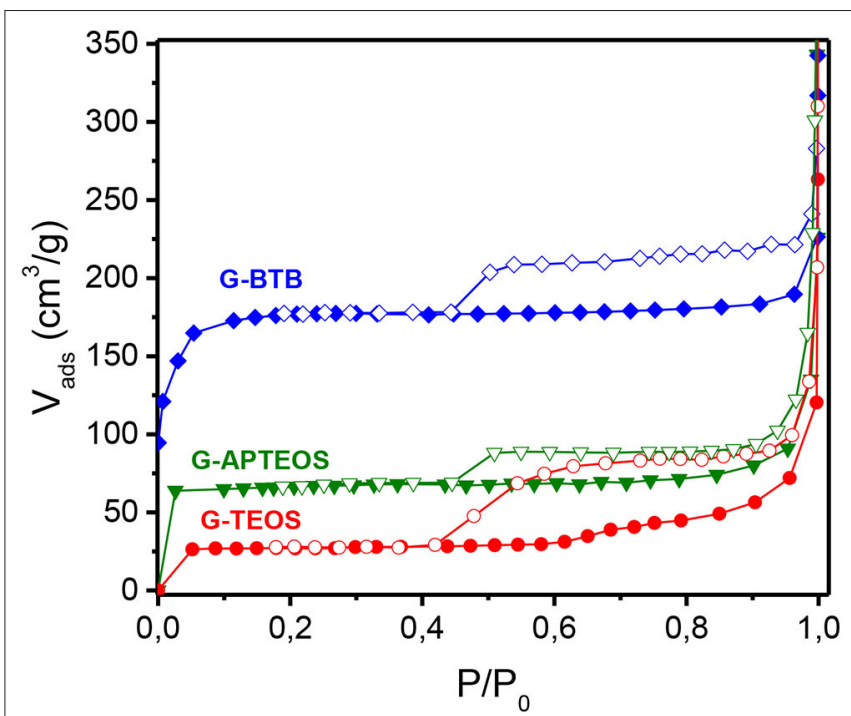

FIGURE 6 | $\mathrm{N}_{2}$ adsorption (full symbols)-desorption (empty symbols) isotherms at $77 \mathrm{~K}$ of heterostructures prepared by silylation of organo-modified graphene oxide with either 1,4-Bis(triethoxysilyl)-benzene, or with

(3-aminopropyl)triethoxysilane, or with tetraethylorthosilicate, and calcinated to give G-BTB, G-APTEOS and G-TEOS.

\section{Characterization Techniques}

The powder X-ray diffraction (XRD) patterns were collected on a D8 Advanced Bruker diffractometer with a $\mathrm{Cu} \mathrm{K}_{\alpha} \mathrm{X}$ ray source $(40 \mathrm{kV}, 40 \mathrm{~mA})$ and a secondary beam graphite monochromator. The patterns were recorded in a $2 \theta$ range from 2 to $40^{\circ}$, in steps of $0.02^{\circ}$ and with a counting time of $2 \mathrm{~s}$ per step. Fourier transform infrared (FTIR) spectra in the range $400-4000 \mathrm{~cm}^{-1}$ were measured with a Shimadzu FT-IR 8400 spectrometer equipped with a deuterated triglycine sulfate (DTGS) detector. Each spectrum was the average of 64 scans collected at $2 \mathrm{~cm}^{-1}$ resolution. Samples were in the form of $\mathrm{KBr}$ pellets containing ca. $2 \mathrm{wt} \%$ sample. X-ray Photoelectron Spectroscopy (XPS) analysis was performed using a Surface Science SSX-100 ESCA instrument with a monochromatic $\mathrm{Al} \mathrm{K \alpha}$ $\mathrm{X}$-ray source $(\mathrm{h} \nu=1486.6 \mathrm{eV})$. The pressure in the measurement chamber was maintained at $1 \times 10^{-9}$ mbar during data acquisition. The electron take-off angle with respect to the surface normal was $37^{\circ}$. The XPS data were acquired by using a spot size of $1,000 \mu \mathrm{m}$ in diameter and the energy resolution was $1.3 \mathrm{eV}$ for both the survey spectra and the detailed spectra of the $\mathrm{C} 1 s, \mathrm{O} 1 s$, and $\mathrm{Si} 2 p$ core level regions. Binding energies are reported $\pm 0.1 \mathrm{eV}$ and referenced to the $\mathrm{C} 1 \mathrm{~s}$ photoemission peak centered at a binding energy of $284.8 \mathrm{eV}$ (Moulder et al., 1995). All XPS spectra were analyzed using the least-squares curve-fitting program Winspec (developed at LISE laboratory of the University of Namur, Belgium). Deconvolution of the spectra included a Shirley baseline (Shirley, 1972) subtraction and fitting with a minimum number of peaks consistent with the chemical structure of the sample, taking into account the experimental resolution. The profile of the peaks was taken as a convolution of Gaussian and Lorentzian functions. The 

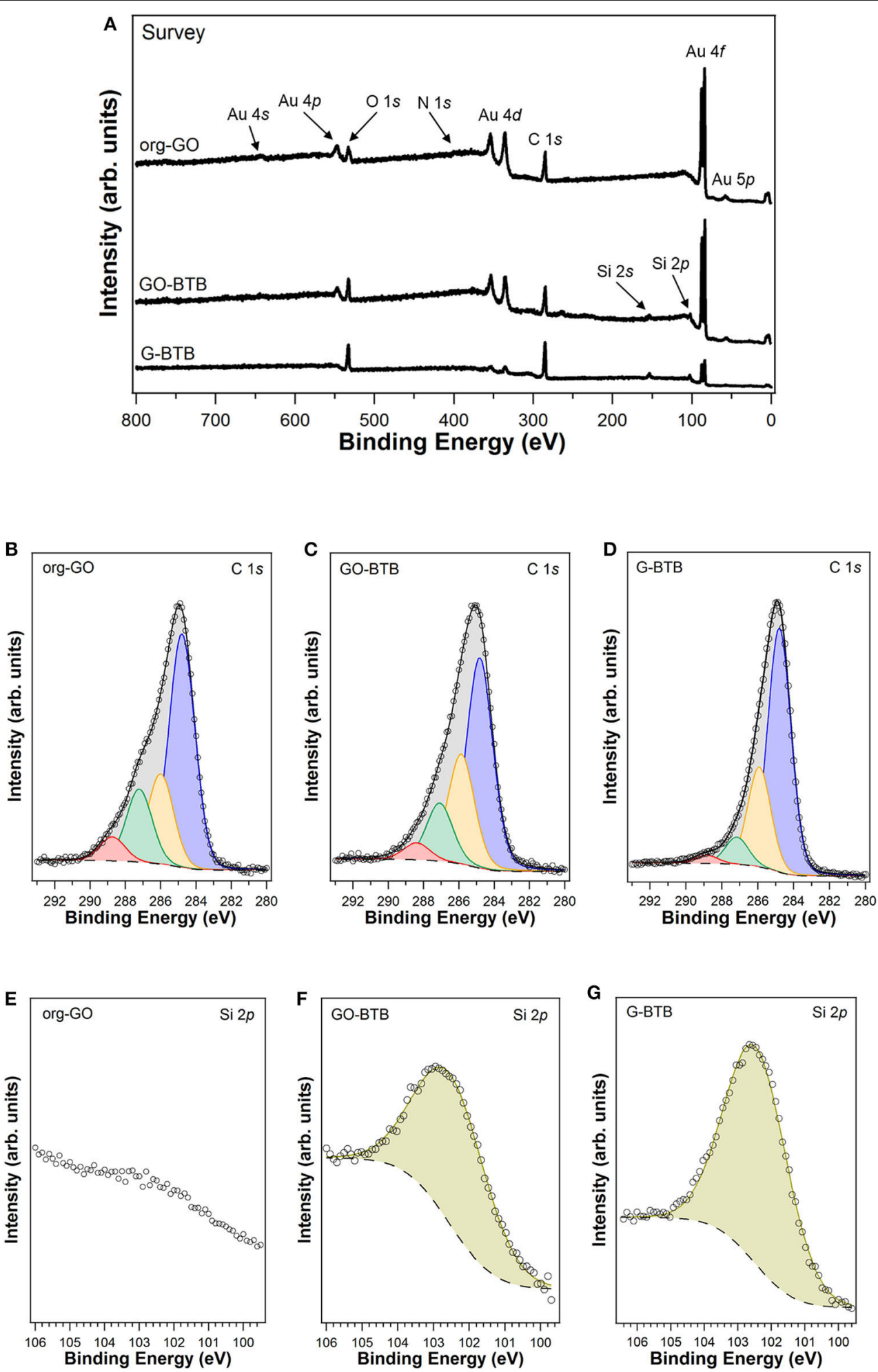

FIGURE 7 | Survey scan [top panel (A)], C1s (B-D) and Si2p (E-G) XPS spectra of graphene oxide intercalated with dodecylamine before (org-GO), after silylation with 1,4-Bis(triethoxysilyl)-benzene (GO-BTB) and after subsequent calcination (G-BTB) (dots) and corresponding fits (full lines); for the differently colored peaks see text.

uncertainty in the peak intensity determination is $2 \%$ for all core levels reported. For the measurements, evaporated polycrystalline gold films supported on mica (grade V-1, TED
PELLA), prepared by sublimation of $99.99 \%$ gold (Schöne Edelmetaal B.V.) as detailed in Mendoza et al. (2007) were used as substrates. Thermogravimetric (TGA) and differential 
TABLE 1 | Binding energies and percentages indicating how much the component contributes to the total C1s intensity; these percentages indicate the relative amounts of carbon atoms involved in each type of bond as deduced from the XPS measurements.

\begin{tabular}{|c|c|c|c|c|}
\hline Carbon species & B.E. (eV) & Sample name and \% & Sample name and \% & Sample name and \% \\
\hline $\mathrm{C}-\mathrm{Si}$ & 283.8 & - & - & GO-APTEOS 5.6 \\
\hline $\mathrm{C}-\mathrm{C} / \mathrm{C}=\mathrm{C}$ & 284.8 & org-GO 55.5 GO-BTB 53.2 G-BTB 70.4 & GO-TEOS 40.7 & GO-APTEOS 40.3 \\
\hline $\mathrm{C}-\mathrm{O} / \mathrm{C}-\mathrm{N}$ & $285.9-286.2$ & org-GO 21.5 GO-BTB 27.8 & GO-TEOS 30.5 & GO-APTEOS 35.4 \\
\hline $\mathrm{C}-\mathrm{O}$ & 285.9 & G-BTB 21.2 & - & - \\
\hline $\mathrm{C}=\mathrm{O} / \mathrm{C}-\mathrm{O}-\mathrm{C}$ & $287.1-287.4$ & org-GO 17.3 GO-BTB 14.7 G-BTB 6.3 & GO-TEOS 21 & GO-APTEOS 14 \\
\hline $\mathrm{COOH}$ & 288.4-288.9 & org-GO 5.7 GO-BTB 4.3 G-BTB 2.1 & GO-TEOS 7.8 & GO-APTEOS 4.7 \\
\hline
\end{tabular}

thermal (DTA) analyses were performed using a Perkin Elmer Pyris Diamond TG/DTA. Samples of approximately $5 \mathrm{mg}$ were heated in air from 25 to $850{ }^{\circ} \mathrm{C}$, at a rate of $5^{\circ} \mathrm{C} / \mathrm{min}$. The nitrogen adsorption-desorption isotherms were measured at $77 \mathrm{~K}$ on a Sorptomatic 1990 Thermo Finnigan porosimeter. Surface area values were determined by the Brunauer-EmmettTeller (BET) method. The surface morphology of the materials was studied using a JEOL JSM-7401F field emission scanning electron microscope (FE-SEM). A low acceleration voltage was applied $(\sim 2 \mathrm{kV})$, and the working distance was set to $3 \mathrm{~mm}$. A powder sample was mounted onto the round brass substrate using double-coated conductive carbon tape. $\mathrm{CO}_{2}$ adsorption isotherms at 0,10 , and $20{ }^{\circ} \mathrm{C}$ were measured on an Intelligent Gravimetric Analyser (IGA-Hiden Ltd.). Before exposure to $\mathrm{CO}_{2}$ the samples were outgassed overnight in $250^{\circ} \mathrm{C}$ under high vacuum $\left(10^{-8}\right.$ mbar $)$ until the mass was observed to remain constant.

\section{RESULTS AND DISCUSSION}

The XRD patterns of the parent materials and of the final hybrids after the sol-gel modification are shown in Figure 2. The XRD pattern of pristine graphite shows the characteristic peak corresponding to the 002 reflection of graphite at $26.6^{\circ}$, which translates into a basal spacing of $3.3 \AA$. This peak disappeared after the oxidation process, when the 001 reflection peak is found at $12.0^{\circ}$, corresponding to a basal spacing of $7.3 \AA$, characteristic of layered GO (Enotiadis et al., 2012). In fact, due to its hydrophilicity, GO exhibits one-dimensional swelling and can exhibit basal spacings between 6.1 and $11 \AA$, depending on the amount of water adsorbed (Dekany et al., 1998). After exposure of GO to dodecylamine the 001 reflection moved to even lower angles, attesting to an increase of the $\mathrm{d}_{001}$ spacing of $\mathrm{GO}$ and hence to the successful insertion of the guest molecules in the interlayer galleries. More specifically, the basal spacing, $\mathrm{d}_{001}$, of the org-GO shifts to $18.4 \AA$ corresponding to an interlayer separation of $\Delta=18.4-6.1=12.3 \AA$, where $6.1 \AA$ is the thickness of the GO monolayer (Dekany et al., 1998). Through the organo-modification, the interlayer space of the GO becomes more accessible and readily modifiable with the silica precursors.

After the sol-gel reaction with silica alkoxides, the 001 reflection of the final hybrid materials is shifted to lower angles than for org-GO, confirming the successful expansion of the interlayer space and suggesting the formation of a silica network for each precursor. When org-GO was treated with the TEOS (GO-TEOS), the $\mathrm{d}$ spacing was calculated to amount to $\sim 24.5 \AA$ and even larger d values (26-27 $\AA$ ) were achieved for APTEOS and BTB. The diffraction peak in this case is broader as a result of a distribution of different conformations. These larger values may be attributed to steric effects from the amino-terminated alkyl chains or phenylene ring anchored/bridged by silane centers of APTEOS and BTB, respectively.

Figure 3 displays the XRD patterns of the silica-GO heterostructures obtained after calcination of GO-BTB, GO-TEOS and GO-APTEOS at $370^{\circ} \mathrm{C}$. For all hybrid heterostructures, the 001 reflection at lower angles $\left(2-10^{\circ}\right)$ is not clearly identifiable but very broad features are observed. This indicates that the graphene layers have lost their ability to stack and proves that the silica-GO heterostructures are in an exfoliated form due to the violent reduction of graphene oxide upon heating (Zhang et al., 2013).

FTIR and XPS spectroscopies can provide additional information on the elemental composition and the type of chemical bonds present in the final hybrid heterostructures. Figure 4 compares the FTIR spectra of GO-BTB before and after (G-BTB) the calcination treatment with those of graphite, pristine (GO) and organo-modified graphene oxide (org-GO). Compared to that of GO, the spectrum of org-GO shows two additional bands at 2847 and $2919 \mathrm{~cm}^{-1}$ associated with the asymmetric and symmetric stretching vibrations of $\mathrm{CH}_{2}$ groups as well as a band at $1450 \mathrm{~cm}^{-1}$ due to the vibrations of the N-H bond of the amino group molecules. These spectral signatures clearly serve as evidence for the presence of dodecylamine in org-GO and thereby testify to the success of the organo-modification.

The spectra of the heterostructure obtained by reaction with the silica precursor BTB exhibit a set of new peaks before (GOBTB) and after calcination (G-BTB), which can be attributed to vibrations of the silicate matrix: the peaks at $520 \mathrm{~cm}^{-1}, 1065$ and $1151 \mathrm{~cm}^{-1}$ are due to $\mathrm{Si}-\mathrm{O}-\mathrm{Si}$ vibrations, while the ones at $690 \mathrm{~cm}^{-1}$ and $950 \mathrm{~cm}^{-1}$ are assigned to the vibrations of $\mathrm{O}-\mathrm{Si}$-O. Similar spectral signatures were also found for the other heterostructures (GO-APTEOS, GO-TEOS, and G-APTEOS, GTEOS, see supporting information, Figure S1) confirming the presence of silica networks in all three heterostructures before and after calcination. For the BTB precursor, two new peaks at 1396 and $3059 \mathrm{~cm}^{-1}$ corresponding to the vibrations of the double bond $\mathrm{C}=\mathrm{C}$ and $\mathrm{C}-\mathrm{H}$ of the phenyl rings appear in the GO-BTB spectrum. The characteristic bands of the intercalated 

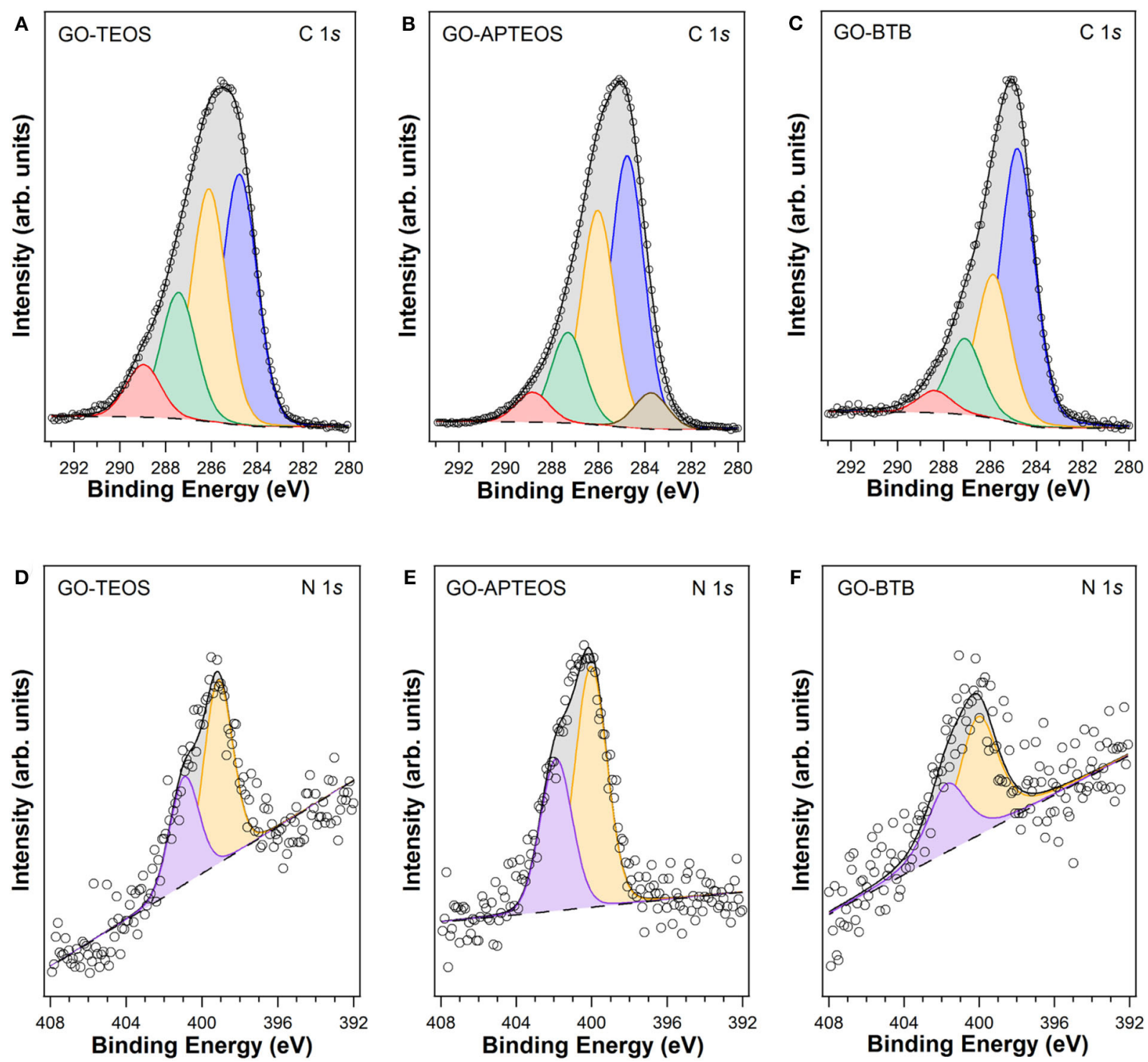

FIGURE 8 |XPS spectra of the C1s (A-C) and N1s (D-F) core level regions of heterostructures prepared by silylation of organo-modified graphene oxide with 1,4-Bis(triethoxysilyl)-benzene (GO-BTB), with (3-aminopropyl)triethoxysilane (GO-APTEOS) and with tetraethylorthosilicate (GO-TEOS) (dots) and corresponding fits (full lines); for the differently colored peaks see text.

dodecylamine identified in the spectrum of org-GO $(2847,2919$ and $1483 \mathrm{~cm}^{-1}$ ) are absent from the spectrum of G-BTB, pointing to the successful removal of the surfactant molecules and hence give a first hint that porous silica-GO heterostructures developed during calcination.

The relative amount of silicon oxide in the three porous structures can be deduced by TGA; Figure 5 presents the results for all three heterostructures. The initial, relatively small weight loss up to about $250{ }^{\circ} \mathrm{C}$ is due to the removal of the adsorbed water and of hydroxy, epoxy and carboxyl groups present in the graphene oxide layers (Bourlinos et al., 2003). When the temperature increases to $450^{\circ} \mathrm{C}$, combustion of dodecylamine takes place (Kooli et al., 2016), while in the temperature range between 500 and $700{ }^{\circ} \mathrm{C}$, the loss mass attests to the combustion of the graphene layers (Jeong et al., 2009) as well as to the dehydroxylation of the silica networks (Ek et al., 2001). The residual mass of $8 \%$ for GO-TEOS, $30 \%$ for GO-APTEOS and $40 \%$ for GO-BTB, corresponds to silicon oxide in each case. These percentages can be explained by the chemical structure of each silica precursor: for APTEOS and BTB alkoxides a larger amount of silica precursor was activated/reacted to give GO-APTEOS and GO-BTB than for the modification with TEOS, which does not have any functional group favoring bonding.

To determine the surface area of the heterostructures after calcination (G-BTB, G-APTEOS and G-TEOS), we monitored $\mathrm{N}_{2}$ adsorption and desorption at $77 \mathrm{~K}$. Figure 6 shows the isotherms obtained, all characterized by an H4 hysteresis loop (based on 

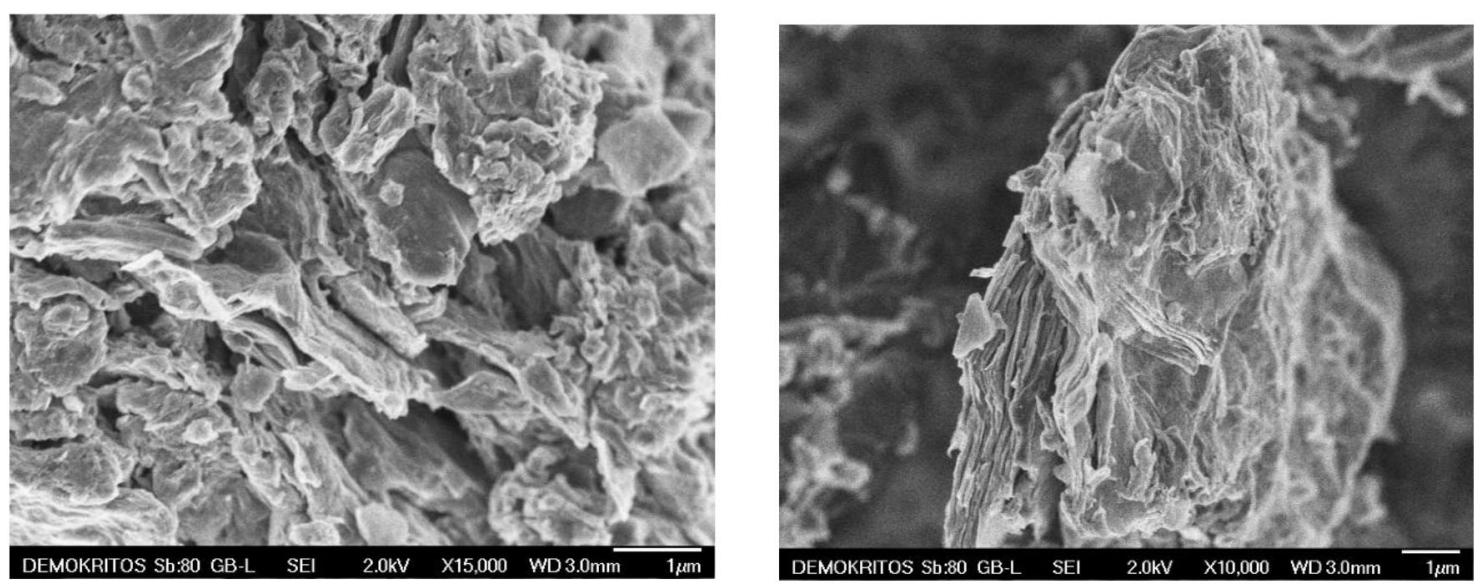

FIGURE 9 | SEM images of the heterostructure prepared by silylation of organo-modified graphene oxide with 1,4-Bis(triethoxysilyl)-benzene, and calcinated to give G-BTB.

IUPAC classification) (Sing et al., 1985; Kalantzopoulos et al., 2014), with a shape typical of slit-shaped pores (Letaïef et al., 2006; Qian et al., 2008). At low relative pressures $\left(\mathrm{P} / \mathrm{P}_{0}<0.01\right)$, the G-BTB heterostructure shows the highest $\mathrm{N}_{2}$ adsorption, which indicates that a significant amount of micropores is accessible after the creation of the silica network. For this system the BET surface area was calculated to be $576 \mathrm{~m}^{2} / \mathrm{g}$, or more than twice that of G-APTEOS $\left(227 \mathrm{~m}^{2} / \mathrm{g}\right)$ and more than 20 times that of G-TEOS $\left(27 \mathrm{~m}^{2} / \mathrm{g}\right)$. Note that for all three heterostructures the final increase of the $\mathrm{N}_{2}$ uptake at relative pressures above 0.95 is attributed to adsorption on the external surface and/or the surface of macropores.

Hence, we followed all modification steps leading to the G-BTB sample by X-ray photoelectron spectroscopy. XPS survey scan of the G-BTB is shown in Figure 7A (top panel). All the expected elements are observed and there is no indication of presence of any kind of contaminant. $\mathrm{C}$ and $\mathrm{O}$ are the main constituents of the sample, while the Au signature is ascribed to the gold surface where the sample was deposited. The C:Si ratio amounts to $6.8 \pm 0.6$, which is comparable with the C:Si ratio before the calcination step, and confirms the presence of BTB molecules in the GO/dodecylamine matrix.

The C1s spectrum of org-GO in Figure 7B shows an asymmetrical peak, which requires four components to obtain a good fit; the main component at a binding energy of $284.8 \mathrm{eV}$ (marked in blue in Figure 7B) can be ascribed to $\mathrm{C}-\mathrm{C} / \mathrm{C}=\mathrm{C}$ species, while the other peaks located at $286.0 \mathrm{eV}, 287.2$ and $288.7 \mathrm{eV}$ can be assigned to $\mathrm{C}-\mathrm{O} / \mathrm{C}-\mathrm{N}$ (yellow), $\mathrm{C}=\mathrm{O} / \mathrm{C}-\mathrm{O}-\mathrm{C}$ (green), $\mathrm{COOH}$ (light red) bonds, respectively (Spyrou et al., 2015; Stathi et al., 2015). The same four components also contribute to the $\mathrm{C} 1 \mathrm{~s}$ line in the cases of GO-BTB (see Figure 7C) and of G-BTB (see Figure 7D). All percentages indicating the relative amounts of carbon atoms involved in each type of bond as deduced from the XPS measurements for org-GO, GO-BTB and G-BTB are shown in Table 1. The O1s core levels of all the samples are shown in the Supporting Information (see Figure S2). The corresponding $\mathrm{Si} 2 p$ lines of GO-BTB and G-BTB in Figures 7F, G are centered at $102.5 \mathrm{eV}$, a binding energy

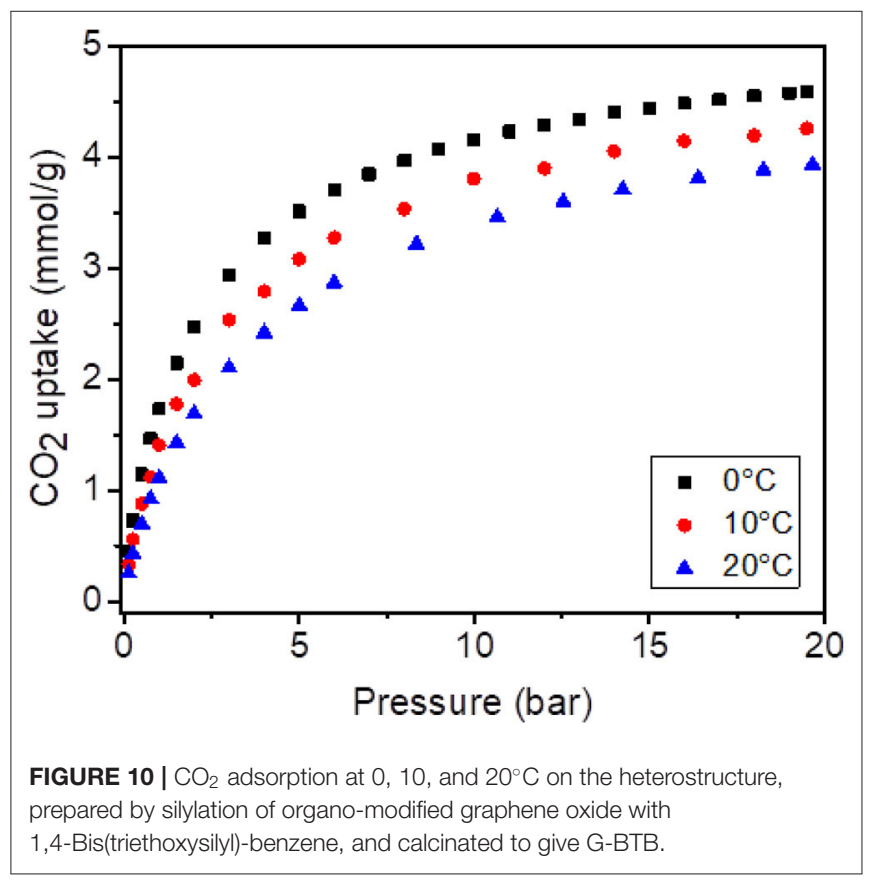

typical of Si-O/Si-O-C species (Kaur et al., 2016). As expected no $\mathrm{Si}$ signal is found for org-GO (Figure 7E). In addition, the $\mathrm{Si} 2 s$ core level spectrum was also acquired to corroborate the presence of only one chemical species of silicon in both samples (see Figure S3 in the Supporting Information). The Si-O-C component is difficult to identify in the $\mathrm{C} 1 s$ peak because of the strong $\mathrm{C}-\mathrm{C} / \mathrm{C}=\mathrm{C}$ and $\mathrm{C}-\mathrm{O} / \mathrm{C}-\mathrm{N}$ signals (Avila et al., 2001).

The presence of the other two precursors employed in the sol-gel synthesis, which lead to GO-TEOS and GOAPTEOS is also confirmed by $\mathrm{X}$-Ray photoelectron spectroscopy. The C1s and N1s XPS spectra are depicted in Figure 8. The $\mathrm{C} 1 \mathrm{~s}$ photoemission spectra of GO-TEOS (Figure 8A) and GO-APTEOS (Figure $\mathbf{8 B}$ ) are similar to GO-BTB before calcination, discussed above and shown again for comparison 
(Figure 8C). Table 1 summarizes the results of the fits in terms of binding energies, attribution of the various components and relative contribution to the total $\mathrm{C} 1 s$ intensity. Since XPS is a quantitative technique, these percentages indicate the relative amounts of carbon atoms involved in each type of bond.

The presence of nitrogen from the intercalated dodecylamine for GO-TEOS, GO-APTEOS and GO-BTB is confirmed by the N1s photoemission lines, shown in Figures 8D-F; note that its position in binding energy at approximately $400.0 \mathrm{eV}$ (yellow in Figure 8) indicates that there is no $\mathrm{C}-\mathrm{N}-\mathrm{C}$ bond, which would give rise to a spectral signature at lower binding energies, but that the amines prefer to bind electrostatically with the oxygen groups of the graphene oxide (Cecchet et al., 2003). The peak at higher binding energies for all hybrid materials (lilac colored in Figure 8) arises from protonated amines that may interact as well with the oxygen functional groups of $\mathrm{GO}(\mathrm{C}-\mathrm{OH}$ and $\mathrm{C}(\mathrm{O}) \mathrm{O})$. In the case of GO-APTEOS there is also the contribution from the amine end groups of APTEOS, which do not participate in the sol-gel reaction.

From the areas of $\mathrm{C} 1 s$ and $\mathrm{Si} 2 p$ lines in the XPS spectra, we calculated the $\mathrm{C}$ to $\mathrm{Si}$ ratio for all three heterostructures, taking into account the sensitivity factors for each element. GOBTB ( $\mathrm{C}: \mathrm{Si}=6.8 \pm 0.5)$ contains a higher amount of $\mathrm{Si}$ than GOAPTEOS (C:Si=65.3 \pm 0.9 ), and GO-TEOS ( $: \mathrm{Si}=101.4 \pm 4.1$ ) ranks lowest in Si content, in agreement with the TGA results discussed above.

Representative scanning electron micrographs of G-BTB are shown in Figure 9. Both SEM images (a) and (b) reveal the layered nature of the porous hybrid heterostructure, confirming the high degree of exfoliation of the GO after silylation and calcination.

The $\mathrm{CO}_{2}$ capture performance of G-BTB was studied at three different temperatures $\left(0,10\right.$, and $\left.20^{\circ} \mathrm{C}\right)$ and for different pressures. The results are presented in Figure 10. From these data one deduces $\mathrm{CO}_{2}$ adsorption capacities of $3.5 \mathrm{mmol} / \mathrm{g}$ at 5 bar and of $4.5 \mathrm{mmol} / \mathrm{g}$ at 17 bar and $0{ }^{\circ} \mathrm{C}$. At higher temperatures the capacity is slightly lower, which is probably because the kinetic energy of the $\mathrm{CO}_{2}$ gas molecules is higher and hence the molecules desorb more easily (lower sticking coefficient). Such high values agree with the high BET surface area and the presence of microporosity. Furthermore, the $\mathrm{CO}_{2}$ adsorption capacity is comparable with that of other graphenebased porous materials with higher surface areas, measured under the same conditions (Chowdhury and Balasubramanian, 2016) This implies that there is potential for achieving even higher values with further structural optimization. Thus, GBTB appears to be a very interesting candidate as $\mathrm{CO}_{2}$ storage material, which combines the properties of graphene with the very high porosity of silica resulting from the sol-gel procedure.

\section{CONCLUSIONS}

Graphene-based porous heterostructures were produced by combining organo-modified graphene oxide with three different organo-silica precursors through sol-gel reactions. After onestep silylation, the interlayer space increased for all the heterostructures compared to organo-modified GO and was maximum for 1,4-Bis(triethoxysilyl)-benzene due to steric effects. FTIR and XPS spectroscopies gave evidence for the reduction of graphene oxide to graphene after calcination of the heterostructures and confirmed the presence of silica oxide. Thermogravimetric analysis allowed to evaluate the degree of silylation for each silica precursor, and to identify 1,4Bis(triethoxysilyl)-benzene (with the bridged phenyl group) as the one giving the highest yield. Thermal treatment is necessary to obtain highly porous materials with sponge-like structures, characterized by a BET surface area of $550 \mathrm{~m}^{2} / \mathrm{g}$ in the case of G-BTB. The latter heterostructure was found to have a high $\mathrm{CO}_{2}$ adsorption capacity of $3.5 \mathrm{mmol} / \mathrm{g}$ at 5 bar and $0{ }^{\circ} \mathrm{C}$, which is promising for further consideration as $\mathrm{CO}_{2}$ storage material.

\section{DATA AVAILABILITY STATEMENT}

The raw data supporting the conclusions of this article will be made available by the authors, without undue reservation.

\section{AUTHOR CONTRIBUTIONS}

AE, DG, and PR designed the experiments and finalized the manuscript. ET and ED performed the synthesis, characterization, and data analysis. EM and EMA participated in the synthesis of the materials. KS and ODL conducted the XPS measurements and analyzed the data. ET, ED, and KS wrote the manuscript. LB performed the SEM measurements. AS performed the $\mathrm{CO}_{2}$ measurements. All authors contributed to manuscript revision, read, and approved the submitted version.

\section{FUNDING}

ET was financially supported by the Hellenic Foundation for Research and Innovation (HFRI) and the General Secretariat for Research and Technology (GSRT), under the HFRI PhD Fellowship grant (GA. No. 1829) and by the Ubbo Emmius programme of the University of Groningen.

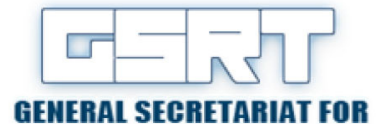

RESEARCH AND TECHNOLOGY
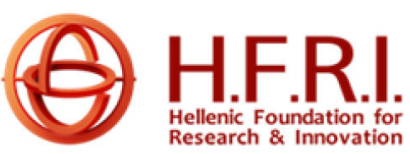

AE gratefully acknowledges financial support by the program of Industrial Scholarships of the Stavros Niarchos Foundation. Partial financial support also came from the Advanced Materials research program of the Zernike National Research Center under the Bonus Incentive Scheme (BIS) of the Dutch Ministry for Education, Culture and Science.

\section{SUPPLEMENTARY MATERIAL}

The Supplementary Material for this article can be found online at: https://www.frontiersin.org/articles/10.3389/fchem. 2020.564838/full\#supplementary-material 


\section{REFERENCES}

Avila, A., Montero, I., Galán, L., Ripalda, J. M., and Levy, R. (2001). Behavior of oxygen doped $\mathrm{SiC}$ thin films: an $\mathrm{x}$-ray photoelectron spectroscopy study. J. Appl. Phys. 89, 212-216. doi: 10.1063/1.1332796

Balomenou, G., Stathi, P., Enotiadis, A., Gournis, D., and Deligiannakis, Y. (2008). Physicochemical study of amino-functionalized organosilicon cubes intercalated in montmorillonite clay: H-binding and metal uptake. J. Colloid Interface Sci 325, 74-83. doi: 10.1016/j.jcis.2008.04.072

Banda, H., Périé, S., Daffos, B., Taberna, P.-L., Dubois, L., Crosnier, O., et al. (2019). Sparsely pillared graphene materials for high-performance supercapacitors: improving ion transport and storage capacity. ACS Nano 13, 1443-1453. doi: 10.1021/acsnano.8b07102

Bourlinos, A. B., Gournis, D., Petridis, D., Szabo, T., Szeri, A., and Dekany, I. (2003). Graphite oxide: chemical reduction to graphite and surface modification with primary aliphatic amines and amino acids. Langmuir 19, 6050-6055. doi: 10.1021/la026525h

Cecchet, F., Pilling, M., Hevesi, L., Schergna, S., Wong, J. K. Y., Clarkson, G. J., et al. (2003). Grafting of benzylic amide macrocycles onto acid-terminated self-assembled monolayers studied by XPS, RAIRS, and contact angle measurements. J. Phys. Chem. B 107, 10863-10872. doi: 10.1021/jp022380b

Chowdhury, S., and Balasubramanian, R. (2016). Three-dimensional graphenebased porous adsorbents for postcombustion $\mathrm{CO}_{2}$ capture. Ind. Eng. Chem. Res. 55, 7906-7916. doi: 10.1021/acs.iecr.5b04052

Dekany, I., Kruger-Grasser, R., and Weiss, A. (1998). Selective liquid sorption properties of hydrophobized graphite oxide nanostructures. Colloid Polym. Sci. $276,570-576$.

dos Santos, T.C., and Ronconi, C.M. (2017). Self-assembled 3D mesoporous graphene oxides (MEGOs) as adsorbents and recyclable solids for $\mathrm{CO}_{2}$ and $\mathrm{CH}_{4}$ capture. J. $\mathrm{CO}_{2}$ Utilization 20, 292-300. doi: 10.1016/j.jcou.2017.05.018

Duan, K., Li, L., Hu, Y., and Wang, X. (2017). Pillared graphene as an ultra-high sensitivity mass sensor. Sci. Rep. 7:14012. doi: 10.1038/s41598-017-14182-6

Ek, S., Root, A., Peussa, M., and Niinistö, L. (2001). Determination of the hydroxyl group content in silica by thermogravimetry and a comparison with 1H MAS NMR results. Thermochim. Acta 379, 201-212. doi: 10.1016/S0040-6031(01)00618-9

Enotiadis, A., Angjeli, K., Baldino, N., Nicotera, I., and Gournis, D. (2012). Graphene-based nafion nanocomposite membranes: enhanced proton transport and water retention by novel organo-functionalized graphene oxide nanosheets. Small 8, 3338-3349. doi: 10.1002/smll.201200609

Enotiadis, A., Litina, K., Gournis, D., Rangou, S., Avgeropoulos, A., Xidas, P., et al. (2013). Nanocomposites of polystyrene-b-Poly(isoprene)-b-Polystyrene triblock copolymer with clay-carbon nanotube hybrid nanoadditivesm. J. Phys. Chem. B 117, 907-915. doi: 10.1021/jp309361b

Gengler, R. Y. N., Veligura, A., Enotiadis, A., Diamanti, E. K., Gournis, D., Jozsa, C., et al. (2010). Large-yield preparation of high-electronic-quatity graphene by a langmuir-schaefer approach. Small 6, 35-39. doi: 10.1002/smll.200901120

Gil, A., Korili, S. A., and Vicente, M. A. (2008). Recent advances in the control and characterization of the porous structure of pillared clay catalysts. Catalysis Rev. 50, 153-221. doi: 10.1080/01614940802019383

Hu, X., Wang, D.-P., Xia, X.-H., Chen, Y.-X., Liu, H.-B., and Gu, Z.-Q. (2019). Pillared graphene sheets with high-rate performance as anode material for lithium-ion batteries. J. Nanosci. Nanotechnol. 19, 7269-7277. doi: $10.1166 /$ jnn.2019.16623

Huang, A., and Feng, B. (2018). Facile synthesis of PEI-GO@ZIF-8 hybrid material for $\mathrm{CO}_{2}$ capture. Int. J. Hydrogen Energy 43, 2224-2231. doi: 10.1016/j.ijhydene.2017.12.070

Jeong, H.-K., Lee, Y. P., Jin, M. H., Kim, E. S., Bae, J. J., and Lee, Y. H. (2009). Thermal stability of graphite oxide. Chem. Phys. Lett. 470, 255-258. doi: $10.1016 /$ j.cplett.2009.01.050

Kalantzopoulos, G. N., Enotiadis, A., Maccallini, E., Antoniou, M., Dimos, K., Policicchio, A., et al. (2014). Hydrogen storage in ordered and disordered phenylene-bridged mesoporous organosilicas. Int. J. Hydrogen Energy 39, 2104-2114. doi: 10.1016/j.ijhydene.2013. 11.063

Kaur, A., Chahal, P., and Hogan, T. (2016). Selective fabrication of SiC/Si diodes by excimer laser under ambient conditions. IEEE Electron Device Letters 37, 142-145. doi: 10.1109/LED.2015.2508479
Ke, Q., and Wang, J. (2016). Graphene-based materials for supercapacitor electrodes - a review. J. Materiomics 2, 37-54. doi: 10.1016/j.jmat.2016.01.001

Kloprogge, J. T., Duong, L. V., and Frost, R. L. (2005). A review of the synthesis and characterisation of pillared clays and related porous materials for cracking of vegetable oils to produce biofuels. Environ. Geol. 47, 967-981. doi: 10.1007/s00254-005-1226-1

Kooli, F., Liu, Y., Hbaieb, K., and Al-Faze, R. (2016). Characterization and catalytic properties of porous clay heterostructures from zirconium intercalated clay and its pillared derivatives. Microporous Mesoporous Mater. 226, 482-492. doi: 10.1016/j.micromeso.2016.02.025

Letaief, S., Martín-Luengo, M. A., Aranda, P., and Ruiz-Hitzky, E. (2006). A colloidal route for delamination of layered solids: novel porous-clay nanocomposites. Adv. Funct. Mater 16, 401-409. doi: 10.1002/adfm. 200500190

Leung, D. Y. C., Caramanna, G., and Maroto-Valer, M. M. (2014). An overview of current status of carbon dioxide capture and storage technologies. Renew. Sustainable Energy Rev. 39, 426-443. doi: 10.1016/j.rser.2014.07.093

Maruyama, J., Akita, S., Matsuo, Y., and Muramatsu, Y. (2014). Silica-pillared graphene sheets with iron-nitrogen units as an oxygen reduction catalyst. Carbon N. Y 66, 327-333. doi: 10.1016/j.carbon.2013.09.007

Matsuo, Y., Hayashida, A., and Konishi, K. (2015). Porous properties of pillared carbons prepared from the thermal reduction of graphite oxide repeatedly silylated with methyltrichlorosilanes. Front. Mater. 2:21. doi: $10.3389 /$ fmats.2015.00021

Matsuo, Y., Komiya, T., and Sugie, Y. (2009). The effect of alkyl chain length on the structure of pillared carbons prepared by the silylation of graphite oxide with alkyltrichlorosilanes. Carbon 47, 2782-2788. doi: 10.1016/j.carbon.2009.06.021

Matsuo, Y., Komiya, T., and Sugie, Y. (2012a). Preparation of microporous pillared carbons from the silylated graphite oxide prepared by a two-step method. $J$. Phys. Chem. Solids 73, 1424-1427. doi: 10.1016/j.jpcs.2011.10.045

Matsuo, Y., Nishino, Y., Fukutsuka, T., and Sugie, Y. (2007). Introduction of amino groups into the interlayer space of graphite oxide using 3-aminopropylethoxysilanes. Carbon 45, 1384-1390. doi: 10.1016/j.carbon.2007.03.037

Matsuo, Y., Tabata, T., Fukunaga, T., Fukutsuka, T., and Sugie, Y. (2005). Preparation and characterization of silylated graphite oxide. Carbon 43, 2875-2882. doi: 10.1016/j.carbon.2005.06.006

Matsuo, Y., Ueda, S., Konishi, K., Marco-Lozar, J. P., Lozano-Castelló, D., and Cazorla-Amorós, D. (2012b). Pillared carbons consisting of silsesquioxane bridged graphene layers for hydrogen storage materials. Int. J. Hydrogen Energy 37, 10702-10708. doi: 10.1016/j.ijhydene.2012.04.033

Mendoza, S. M., Arfaoui, I., Zanarini, S., Paolucci, F., and Rudolf, P. (2007). Improvements in the characterization of the crystalline structure of acidterminated alkanethiol self-assembled monolayers on $\mathrm{Au}(111)$. Langmuir 23, 582-588. doi: 10.1021/la0605539

Moulder, J. F., Stickle, W. F., and Sobol, P. E. (1995). Handbook of XRay Photoelectron Spectroscopy: A Reference Book of Standard Spectra for Identification and Interpretation of XPS Data. Eden Prairie, Minn.: Physical Electronics, Inc.

National Academies of Sciences and Medicine (2019). Gaseous Carbon Waste Streams Utilization: Status and Research Needs. Washington, DC: The National Academies Press.

Nicotera, I., Enotiadis, A., Angjeli, K., Coppola, L., Ranieri, G. A., and Gournis, D. (2011). Effective improvement of water-retention in nanocomposite membranes using novel organo-modified clays as fillers for high temperature PEMFCs. J. Phys. Chem. B 115, 9087-9097. doi: 10.1021/jp202954g

Novoselov, K. S., Fal' ko, V. I., Colombo, L., Gellert, P. R., Schwab, M. G., and Kim, K. (2012). A roadmap for graphene. Nature 490, 192-200. doi: 10.1038/nature11458

Ohtsuka, K. (1997). Preparation and properties of two-dimensional microporous pillared interlayered solids. Chem. Mater. 9, 2039-2050.

Pedrielli, A., Taioli, S., Garberoglio, G., and Pugno, N. M. (2018). Gas adsorption and dynamics in pillared graphene frameworks. Microporous Mesoporous Mater. 257, 222-231. doi: 10.1016/j.micromeso.2017.08.034

Pokhrel, J., Bhoria, N., Anastasiou, S., Tsoufis, T., Gournis, D., Romanos, G., et al. (2018). $\mathrm{CO}_{2}$ adsorption behavior of amine-functionalized ZIF-8, graphene oxide, and ZIF-8/graphene oxide composites under dry and wet conditions. Microporous Mesoporous Mater. 267, 53-67. doi: 10.1016/j.micromeso.2018.03.012 
Qian, Z., Hu, G., Zhang, S., and Yang, M. (2008). Preparation and characterization of montmorillonite-silica nanocomposites: A sol-gel approach to modifying clay surfaces. Physica B 403, 3231-3238. doi: 10.1016/j.physb.2008.04.008

Rana, S., Maddila, S., Yalagala, K., Maddila, S., and Jonnalagadda, S. B. (2015). Covalent modification of organo-functionalized graphene oxide and its scope as catalyst for one-pot pyrazolo-pyranopyrimidine derivatives. Chem. Open 4, 703-707. doi: 10.1002/open.201500121

Saha, D., and Kienbaum, M. J. (2019). Role of oxygen, nitrogen and sulfur functionalities on the surface of nanoporous carbons in $\mathrm{CO}_{2}$ adsorption: a critical review. Microporous Mesoporous Mater. 287, 29-55. doi: 10.1016/j.micromeso.2019.05.051

Shi, X., Xiao, H., Azarabadi, H., Song, J., Wu, X., Chen, X., et al. (2020). Sorbents for the direct capture of $\mathrm{CO}_{2}$ from ambient air. Angew. Chem. Intern. Edition 59, 6984-7006. doi: 10.1002/anie.201906756

Shirley, D. A. (1972). High-resolution X-ray photoemission spectrum of the valence bands of gold. Phys. Rev. B 5, 4709-4714. doi: 10.1103/PhysRevB. 5.4709

Shrivastava, S., Thomas, S., Sobhan, C. B., and Peterson, G. P. (2018). An experimental investigation of the $\mathrm{CO}_{2}$ adsorption performance of graphene oxide forms. Intern. J. Refrigerat. 96, 179-190. doi: 10.1016/j.ijrefrig.2018.07.014

Sing, K. S. W., Everett, D. H., Haul, R. A. W., Moscou, L., Pierotti, R. A., Rouquerol, J., et al. (1985). Reporting physisorption data for gas solid systems with special reference to the determination of surface-area and porosity (Recommendations 1984). Pure Appl. Chem. 57, 603-619.

Spyrou, K., Calvaresi, M., Diamanti, E. A. K., Tsoufis, T., Gournis, D., Rudolf, P., et al. (2015). Graphite oxide and aromatic amines: size matters. Adv. Funct. Mater. 25, 263-269. doi: 10.1002/adfm.201402622

Stathi, P., Gournis, D., Deligiannakis, Y., and Rudolf, P. (2015). Stabilization of phenolic radicals on graphene oxide: an XPS and EPR study. Langmuir 31, 10508-10516. doi: 10.1021/acs.langmuir.5b01248

Staudenmaier, L. (1898). Verfahren zur darstellung der graphitsaure. Ber. Deut. Chem. Ges. 31, 1481.

Stergiou, D. V., Diamanti, E. K., Gournis, D., and Prodromidis, M. I. (2010). Comparative study of different types of graphenes as electrocatalysts for ascorbic acid. Electrochem. Commun. 12, 1307-1309. doi: 10.1016/j.elecom.2010.07.006

Tonlé, I. K., Diaco, T., Ngameni, E., and Detellier, C. (2007). Nanohybrid kaolinite-based materials obtained from the interlayer grafting of 3aminopropyltriethoxysilane and their potential use as electrochemical sensors. Chem. Mater. 19, 6629-6636. doi: 10.1021/cm702206z

Wahab, M. A., and He, C. (2009). Self-assembly, optical, and mechanical properties of surfactant-directed biphenyl-bridged periodic mesostructured organosilica films with molecular-scale periodicity in the pore walls. Langmuir 25, 832-838. doi: $10.1021 /$ la803192z

Wahab, M. A., Hussain, H., and He, C. (2009). Photoactive perylenediimidebridged silsesquioxane functionalized periodic mesoporous organosilica thin films (PMO-SBA15): synthesis, self-assembly, and photoluminescent and enhanced mechanical properties. Langmuir 25, 4743-4750. doi: 10.1021/la900042g

Wahab, M. A., Imae, I., Kawakami, Y., and Ha, C.-S. (2005). Periodic mesoporous organosilica materials incorporating various organic functional groups: synthesis, structural characterization, and morphology. Chem. Mater. 17, 2165-2174. doi: 10.1021/cm0480059

Wahab, M. A., Imae, I., Kawakami, Y., Kim, I., and Ha, C.-S. (2006). Functionalized periodic mesoporous organosilica fibers with longitudinal pore architectures under basic conditions. Microporous Mesoporous Mater. 92, 201-211. doi: 10.1016/j.micromeso.2005.12.016

Wahab, M. A., Kim, I., and Ha, C.-S. (2004b). Bridged amine-functionalized mesoporous organosilica materials from 1,2-bis(triethoxysilyl)ethane and bis[(3-trimethoxysilyl)propyl]amine. J. Solid State Chem. 177, 3439-3447. doi: 10.1016/j.jssc.2004.05.062

Wahab, M. A., Kim, I. I., and Ha, C.-S. (2004a). Hybrid periodic mesoporous organosilica materials prepared from 1,2-bis(triethoxysilyl)ethane and (3cyanopropyl)triethoxysilane. Microporous Mesoporous Mater. 69, 19-27. doi: 10.1016/j.micromeso.2004.01.001

Yan, J., Liu, J., Fan, Z., Wei, T., and Zhang, L. (2012). High-performance supercapacitor electrodes based on highly corrugated graphene sheets. Carbon 50, 2179-2188. doi: 10.1016/j.carbon.2012.01.028

Yoo, H. D., Park, Y., Ryu, J. H., and Oh, S. M. (2011). Electrochemical activation behaviors studied with graphitic carbon electrodes of different interlayer distance. Electrochim. Acta 56, 9931-9936. doi: 10.1016/j.electacta.2011. 08.117

Zapata, P. A., Belver, C., Quijada, R., Aranda, P., and Ruiz-Hitzky, E. (2013). Silica/clay organo-heterostructures to promote polyethylene-clay nanocomposites by in situ polymerization. Appl. Catalysis A 453, 142-150. doi: 10.1016/j.apcata.2012.12.012

Zhang, C., Lv, W., Xie, X., Tang, D., Liu, C., and Yang, Q.-H. (2013). Towards low temperature thermal exfoliation of graphite oxide for graphene production. Carbon 62, 11-24. doi: 10.1016/j.carbon.2013.05.033

Zhao, G., Jiang, L., He, Y., Li, J., Dong, H., Wang, X., et al. (2011). Sulfonated graphene for persistent aromatic pollutant management. Adv. Mater. 23, 3959-3963. doi: 10.1002/adma.201101007

Conflict of Interest: The authors declare that the research was conducted in the absence of any commercial or financial relationships that could be construed as a potential conflict of interest.

Copyright (C) 2020 Thomou, Diamanti, Enotiadis, Spyrou, Mitsari, Boutsika, Sapalidis, Moretón Alfonsín, De Luca, Gournis and Rudolf. This is an open-access article distributed under the terms of the Creative Commons Attribution License (CC $B Y)$. The use, distribution or reproduction in other forums is permitted, provided the original author(s) and the copyright owner(s) are credited and that the original publication in this journal is cited, in accordance with accepted academic practice. No use, distribution or reproduction is permitted which does not comply with these terms. 